\title{
THE PROJECTION OF THE OLFACTORY EPITHELIUM ON THE OLFACTORY BULB IN THE RABBIT
}

\author{
BY \\ W. E. LE GROS CLARK \\ From the Department of Anatomy, University of Oxford
}

In a previous study (Le Gros Clark and Warwick, 1946) the degeneration of olfactory receptors in the rabbit following complete or partial ablation of the olfactory bulb was examined by reference to sections of the mucosa covering the septal cartilage, stained by Bodian's protargol method. Evidence was advanced to show that this is a true secondary degeneration following interruption of the primary olfactory neurons, and not the result of other factors such as vascular disturbance. It was found that the degeneration of the olfactory receptors occurs with great rapidity, for they undergo almost complete disintegration one week after removal of the bulb. The atrophy of the receptors is associated with a shrinkage in depth of the whole epithelium. The histological picture of the septal epithelium following partial lesions of the bulb incidentally led to the conclusion that there is no definite localized projection of different areas of the olfactory epithelium on to the bulb, for such lesions appeared to lead to a general thinning out of the receptors rather than to localized areas of complete degeneration. Later, evidence was brought forward by Adrian (1950), on the basis of electrophysiological studies, that there is in fact some degree of localized projection, though of a rather diffuse kind. $\mathrm{He}$ further made the interesting suggestion that this may have some importance as a basis for olfactory discrimination. It therefore appeared desirable to investigate the problem further by experimental anatomical methods, without limiting observations to a restricted area of the septal mucosa. As will be seen, this more extended enquiry has provided definite evidence of a general type of localization in the projection of the olfactory epithelium on the olfactory bulb.

\section{Material and Methods}

Partial or complete ablations of the bulb were made in 14 rabbits. Young animals, about 3 months old, were used for the experiments in order to eliminate as far as possible the complications of chronic rhinitis which is liable to affect older laboratory animals. Under nembutal anaesthesia, and with aseptic precautions, the bulb was exposed on one side by removing the frontal bone covering it. The lesions were effected with a fine-bladed scalpel, and in every case the resulting haemorrhage was readily controlled by slight pressure with a pledget of wool. The animals were allowed to survive two to six weeks. The brain and olfactory mucosa were fixed by perfusion through the aorta of $5 \%$ formol saline. The frontal poles of the brain, with the attached remnants of the operated bulb and the normal bulb of the opposite side, were removed, embedded in one block, and cut in serial sections. The latter were stained with haematoxylin and eosin or methylene blue. The whole of that part of the nasal cavity containing the olfactory epithelium was resected and, after decalcification in 5\% nitric acid, serially sectioned. Every fiftieth section was mounted and stained with haematoxylin and eosin.

From the serial sections of the olfactory bulbs the position and extent of the lesion in each experiment were defined. For several reasons, however, this could only be done approximately. In the first place, the operation usually resulted in some distortion of the olfactory bulb (with considerable dilatation of the olfactory ventricle), so that it was not always easy to compare precisely the situation of the destroyed glomeruli with those of the normal bulb of the opposite side. Secondly, the zone of complete destruction was in many cases bordered by glomeruli which, though not directly affected by the lesion, had undergone varying degrees of atrophy consequent on the interruption of their afferent olfactory connexions. The assessment of the extent of this indirect, partial involvement of glomeruli was difficult. Account had also to be taken of secondary involvement due to vascular interruption, and as a result of this factor it was found that lesions which were intended to be small and localized had extended some way beyond the actual site of operation. For this reason, also, in three cases in which relatively large lesions had been made (with the intention of leaving intact small remnants of the glomerular system), it was found that total destruction of the bulb had in fact resulted.

The serial sections of the nasal cavity were used to plot the distribution of the secondary degeneration in the olfactory epithelium. The sections were stained 
with haematoxylin and eosin because of the difficulty of obtaining in decalcified material a satisfactory and consistent demonstration of the olfactory receptors by methods of silver impregnation. The extent of the secondary degeneration was therefore determined indirectly by the degree of reduction in the total depth of the epithelial layer, which (as already noted) accompanies the atrophy and disintegration of the olfactory sensory cells. It is important to note, however, that this criterion of the extent of the retrograde degeneration of the receptors only provides data of an approximate kind, for basides the olfactory sensory elements the epithelium also contains supporting cells and basal cells, which do not themselves undergo degeneration. Further, in those areas, as in the recesses at the attachment of the turbinate processes, where the olfactory epithelium is thinner than elsewhere, and the proportion of supporting and basal cells to olfactory receptors somewhat greater, the reduction in the total depth of the epithelium in relation to the bulbar lesions tends to be correspondingly less. Lastly, with the histological methods employed, it is, of course, not possible to affirm that in areas where the olfactory epithelium appears normal a few scattered receptors may not have undergone atrophy, nor is it possible to state that in areas of severe degeneration scattered receptors may not have survived.

The degree of shrinkage of the epithelium was measured with an ocular micrometer over different parts of the septum and all the turbinate processes, and compared with the normal epithelium at corresponding points in the opposite side of the nasal cavity. In each local region compared, the mean of ten separate measurements was taken. In order to obtain a general indication of the distribution of degeneration of different degrees of severity (complete or almost complete, and partial or slight), two arbitrary grades were selected : (1) severe degeneration, in which the total depth of the epithelium had been reduced by at least $25 \%$ when compared with the normal side, and (2) moderate degeneration, in which the reduction was at least $10 \%$ (Fig. 1). Following total resection of one olfactory bulb, the average reduction in depth of the olfactory epithelium of that side in all areas (as measured on the sections) is $40 \%$. In the first experiments, the animals were allowed to survive six weeks after operation in order to ensure that the maximum resulting degeneration of the olfactory epithelium had occurred. It was subsequently found that, in fact, this occurs by the end of two weeks. This is shown by the following comparisons of the percentage reduction in the depth of the olfactory epithelium in different regions after total ablation of the olfactory bulb in two cases in which the animals were killed two weeks and six weeks respectively after operation :-

\begin{tabular}{|c|c|c|}
\hline Region & $\begin{array}{l}\text { Experiment L. } 2 \\
\text { ( } 2 \text { weeks) }\end{array}$ & $\begin{array}{l}\text { Experiment R.643 } \\
\text { (6 weeks) }\end{array}$ \\
\hline $\begin{array}{cc}\text { Septum } \\
\text { Nasoturbinal } \\
\text { Endoturbinal } & 1 \\
, & 2 \\
\text { ", } & 3 \\
\text { Ectoturbinal } & 1 \\
\text {, } & 2 \\
\text { ", } & 3\end{array}$. & $\begin{array}{l}44 \\
45 \\
42 \\
27 \\
38 \\
30 \\
50 \\
46 \\
38\end{array}$ & $\begin{array}{l}43 \\
45 \\
49 \\
38 \\
36 \\
30 \\
47 \\
30 \\
39\end{array}$ \\
\hline
\end{tabular}

In three experiments with partial lesions of the olfactory bulb the results had to be discarded, because the sections of the nasal cavity showed evidence of rhinitis which made it impossible to define the areas of secondary degeneration of the olfactory epithelium with accuracy.

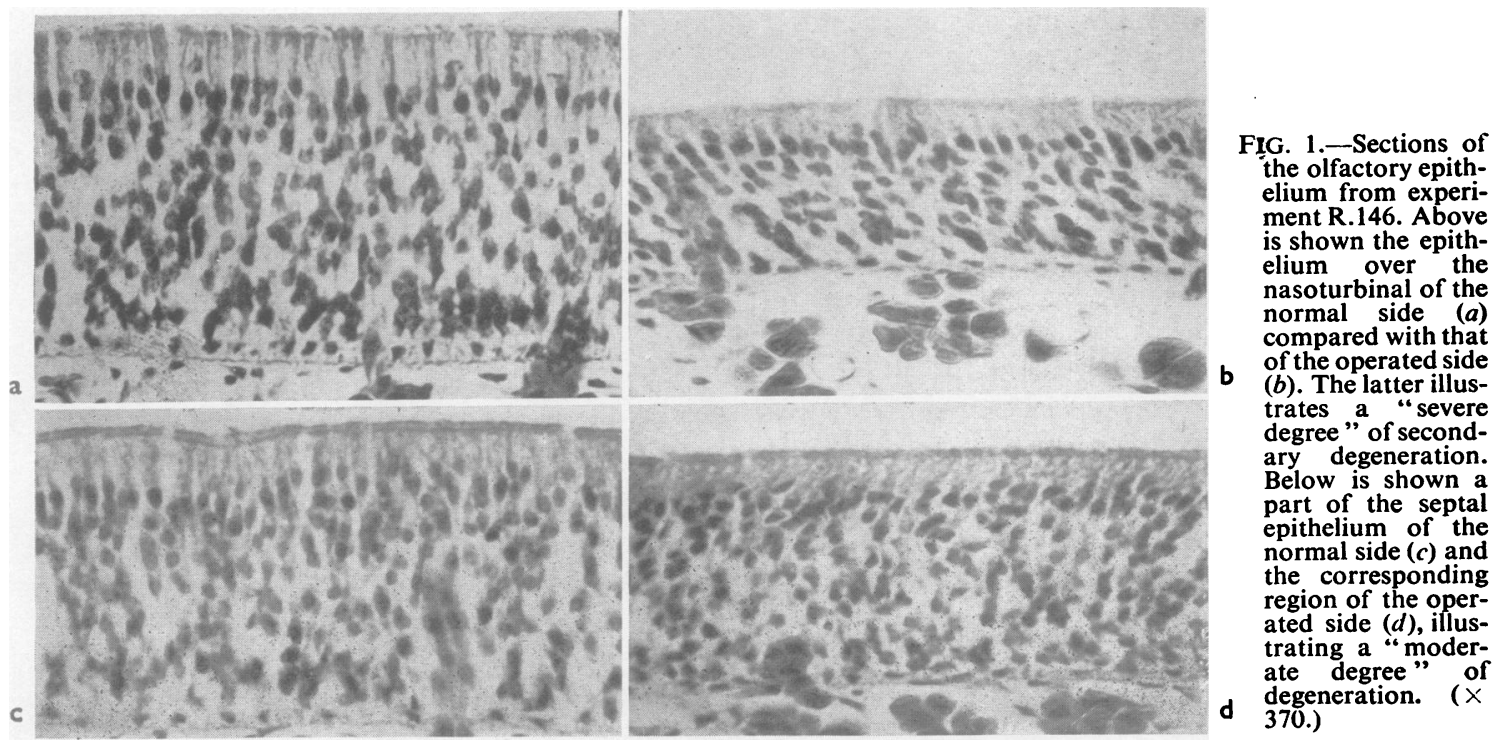




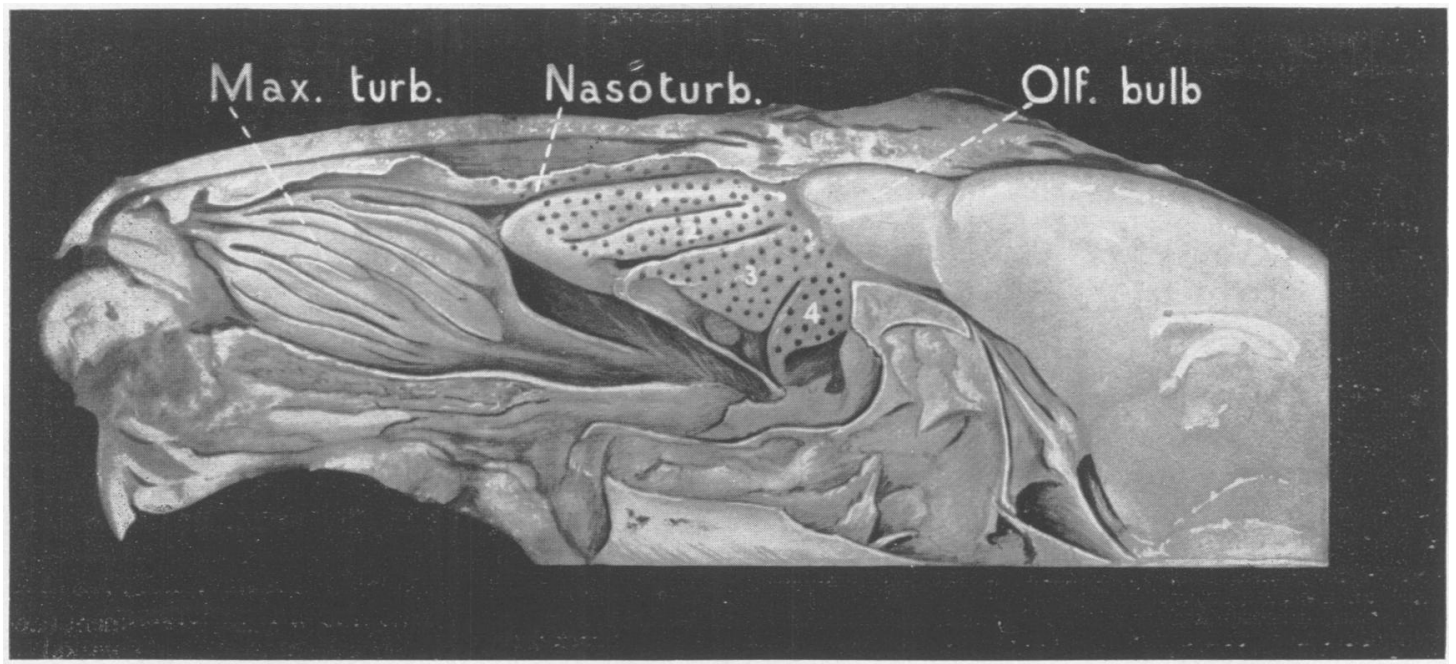

Fig. 2.-Sagittal section of a rabbit's head showing the appearance of the right nasal cavity after removal of the septum. The extent of the olfactory epithelium is indicated by the dotted area. The endoturbinals are numbered $1-4$.

General Topography of the Olfactory Epithelium in the Rabbit

Before recording the results of individual experiments it is desirable to make a brief reference to the topography of the areas of olfactory epithelium in the rabbit. As is well known, in lower mammals this epithelium covers the postero-superior part of the septum and a complicated series of turbinate processes in the lateral part of the nasal cavity. In the rabbit (Fig. 2) there are four endoturbinals (processes of the ethmoid bone) whose free surfaces are apposed to the septum. Above the first (uppermost) endoturbinal is the nasoturbinal which extends forwards on the under surface of the nasal bone. In the roof of the nasal cavity, between the nasoturbinal and the septum, is a narrow recess which will be referred to as the "dorsal recess". Extending laterally between the nasoturbinal and the first endoturbinal is a in each case being shown to the right. large "lateral recess" of the nasal cavity into which project three ectoturbinals (Figs. 3-8) which are here termed the upper, middle, and lower ectoturbinals. The upper and lower are large processes bearing an extensive area of olfactory epithelium. The middle ectoturbinal is a small process attached to the middle of the lateral wall of the lateral recess. Lateral to the third endoturbinal there may be an additional small ectoturbinal.

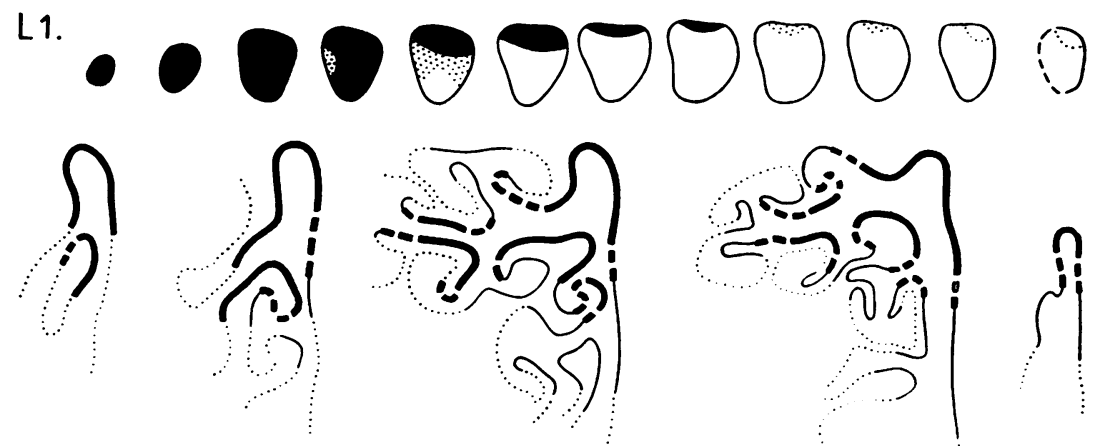

FIG. 3.-Diagram illustrating the extent of the lesion in the olfactory bulb and of the secondary degeneration of the olfactory epithelium in experiment L.1.

In Figs. 3-8 areas of severe degeneration of the olfactory epithelium are indicated by a continuous thick line, and of moderate degeneration by an interrupted thick line. Areas of apparently normal (or nearly normal) epithelium are indicated by a continuous thin line. Dotted lines represent the nonolfactory part of the nasal mucous membrane.

Twelve transverse sections of the olfactory bulb, taken at regular intervals, are represented above. The extent of total destruction is indicated in black and areas of partial involvement in stipple. In all cases the lateral aspect of the bulb is shown to the left. Below are shown, from left to right, outlines of transverse sections taken at regular antero-posterior intervals through the nasal cavity of the affected side, the septal epithelium 
In Figs. 3-8 areas of severe degeneration of the olfactory epithelium are indicated by a continuous thick line, and of moderate degeneration by an interrupted thick line. Areas of apparently normal (or nearly normal) epithelium are indicated by a continuous thin line. Dotted lines represent the non-olfactory

part of the nasal mucous membrane.
Twelve transverse sections of the olfactory bulb, taken at regular intervals, are represented above. The extent of total destruction is indicated in black and areas of partial involvement in stipple. In all cases the lateral aspect of the bulb is shown to the left. Below are shown, from left to right, outlines of transverse sections taken at regular antero-posterior intervals through the nasal cavity of the affected side, the septal epithelium in each case being shown to the right.

\section{Results}

Experiment L.1 (Fig. 3). - At operation, approximately the anterior third of one olfactory bulb was removed and the animal killed five weeks later. Serial sections show that the lesion is fairly well circumscribed but, presumably as the result of vascular interference, a narrow zone of necrosis extends back on the upper surface of the bulb as far as the junction of its middle and posterior thirds. Except in the neighbourhood of the main lesion, the glomerular formations of the remaining part of the bulb appear normal.

Serial sections through the nasal cavity show severe degeneration of the olfactory epithelium extending through the whole extent of its dorsal part. It affects the lining of the dorsal recess, approximately the dorsal half of the septum, the greater part of the nasoturbinal, the dorsal and medial aspects of the first and second endoturbinals, and the opposed surfaces of the upper and lower ectoturbinals. These areas are bordered by fairly wide zones of moderate degeneration. The olfactory epithelium appears to be normal over the lower half of the posterior part of the septum, the greater part of the third endoturbinal, the whole of the fourth endoturbinal, and the middle ectoturbinal. Fig. 3 shows some correspondence between the position of the bulbar lesion and the area of secondary degeneration of the olfactory epithelium, but the correspondence is no more than approximate. The relative extent of this area appears greater in the more anterior sections, but it reaches back to the most posterior part of the nasal cavity. The septal epithelium is affected in part throughout its antero-posterior extent even though the medially situated glomeruli in the posterior half of the bulb are apparently normal. On the other hand, the absence of definite degeneration over the lower half of the posterior part of the septum, and the fourth and most of the third endoturbinals, is presumably related to the fact that, in general, they project on to the lower and caudal parts of the bulb. The unaffected parts of the ectoturbinal epithelium evidently have a similar projection.

Experiment R.146 (Fig. 4).-The intention in this experiment was to place a lesion limited to the lateral half of one olfactory bulb. Serial sections of the bulb show that it was mainly confined to the dorsal surface over the greater extent of the bulb, but there is a considerable cellular infiltration in the lateral surface of the anterior half of the bulb and the glomeruli in this region are grossly shrunken (though only the most dorsally situated are completely destroyed). On the other hand, the medially situated glomeruli appear normal. The olfactory ventricle is greatly distended.

The serial sections of the nasal cavity show severe degeneration of the epithelium lining the dorsal recess, and of that covering the upper part of the septum, the whole length of the nasoturbinal, and the upper surface of the first endoturbinal. A moderate degree of epithelial degeneration is present over the medial part of the upper ectoturbinal and a narrow zone on the upper surface of the second endoturbinal, as well as alongside the margins of the severely degenerated areas. There is a broad relationship between the dorsally situated lesion of the bulb and the limitation of the epithelial degeneration mainly to the upper part of the nasal cavity. On the other hand, the involvement of the laterally situated glomeruli is not reflected in much degeneration of the lateral areas of olfactory epithelium. Compared with the previous experiment, L.1, the epithelial degeneration is not so extensive or severe over the anterior extremities of the first endoturbinal, the nasoturbinal, or the upper ectoturbinal. This is evidently related to the fact that the anterior quarter of the olfactory bulb was left almost intact. In some areas, particularly over the first endoturbinal (Fig. 9), the junction between severely degenerated and apparently normal epithelium is quite abrupt, suggesting that here the regional projection on to the bulb may be rather precise.

Experiment R.634.-A small lesion was made in the frontal half of the dorsal surface of one olfactory bulb, and the animal killed two weeks later. Serial sections show that the lesion is very limited, involving a narrow strip of the dorsal aspect of the bulb and extending back almost to the level of the accessory bulb. It corresponds closely to that of the previous experiment (R.146) but without the involvement of the laterally situated glomeruli. 


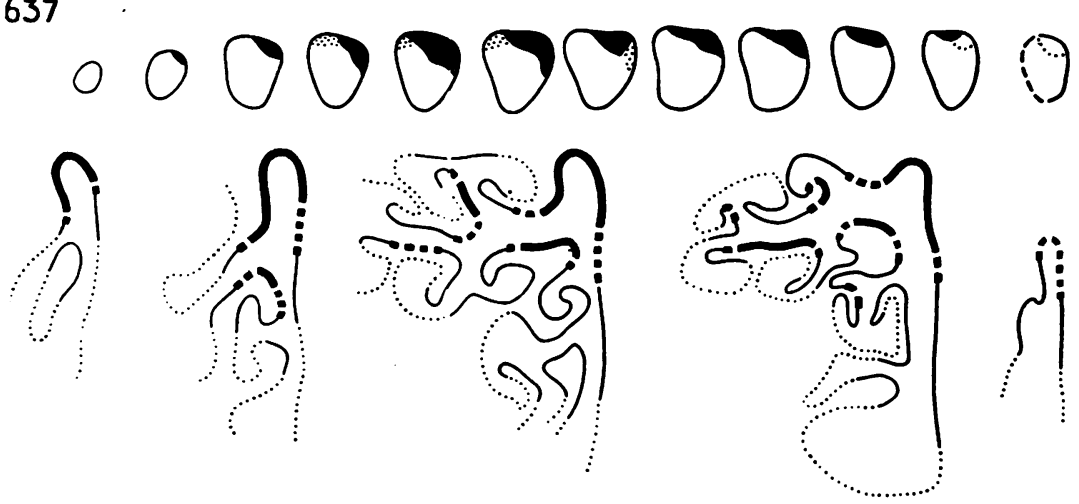

FIG. 5.-Diagram illustrating experiment R.637.

evident degeneration over the whole extent of the lower half of the septum, makes it clear that there is some degree of dorsi-ventral projection from the olfactory epithelium to the olfactory bulb.

Experiment R.638 (Fig. 6).-In this case the lesion was mainly limited to the anterior third of one bulb. The animal was killed two weeks after the operation. The posterior

The serial sections of the olfactory cavity are somewhat imperfect, but they permit a satisfactory delineation of the areas of epithelial degeneration. The distribution of the degeneration conforms closely to that of experiment R.146, except that it extends further down on the septum (particularly anteriorly), is less marked on the upper aspect of the first endoturbinal, and does not appear to involve the second endoturbinal at all. At the most posterior extremity of the nasal cavity there is little evidence of degeneration except for a moderate degree near the upper margin of the septum and the lateral wall of the dorsal recess.

Experiment R.637 (Fig. 5).-A lesion was made on the medial aspect of the anterior half of one bulb. The animal was killed two weeks later. Serial sections show that, probably as the result of vascular damage, a zone of necrosis extends back from the site of the operative lesion on the dorsal aspect of the bulb. They also make it apparent that the lesion had not involved as much of the medial aspect of the glomerular system as had been intended.

Sections of the nasal cavity show that the distribution of the epithelial degeneration is somewhat similar to that of the previous experiments, but rather more extensive. For example, it involves more of the ectoturbinal epithelium even though the laterally situated glomeruli were less affected by the lesion than in experiment R.146. The degeneration of the septal epithelium also extends further down over the more posterior part of the septum. The epithelium covering the second, third, and fourth endoturbinals shows no gross evidence of degenerative change. This, together with the absence of extension of the lesion was found to be considerably less than in any of the four previous experiments. In conformity with this, the retrograde degeneration of the olfactory epithelium is also less extensive, and in the most posterior sections it shows no gross change. Nevertheless, the distribution of the degeneration is considerably wider than would be expected on the basis of a sharply localized topical projection on to the bulb. Over the upper part of the septum in its anterior two-thirds, a corresponding extent of the nasoturbinal, the convexity of the upper ectoturbinal, and the upper surface of the whole length of the first endoturbinal, the olfactory epithelium shows severe degeneration. A moderate degree of degeneration is also present over the upper surface of the lower ectoturbinal and in marginal zones bounding the strips of severe degeneration. It will be noted (Fig. 6) that the epithelium at the summit of the dorsal recess is represented as being not obviously degenerated. However, it is not possible to make any definite statement about this region, because the corresponding olfactory epithelium of the opposite side shows some shrinkage (? of rhinitic origin), which thus precludes a comparison with the operated side. Compared with experiment R.637 the epithelial degeneration extends further forward

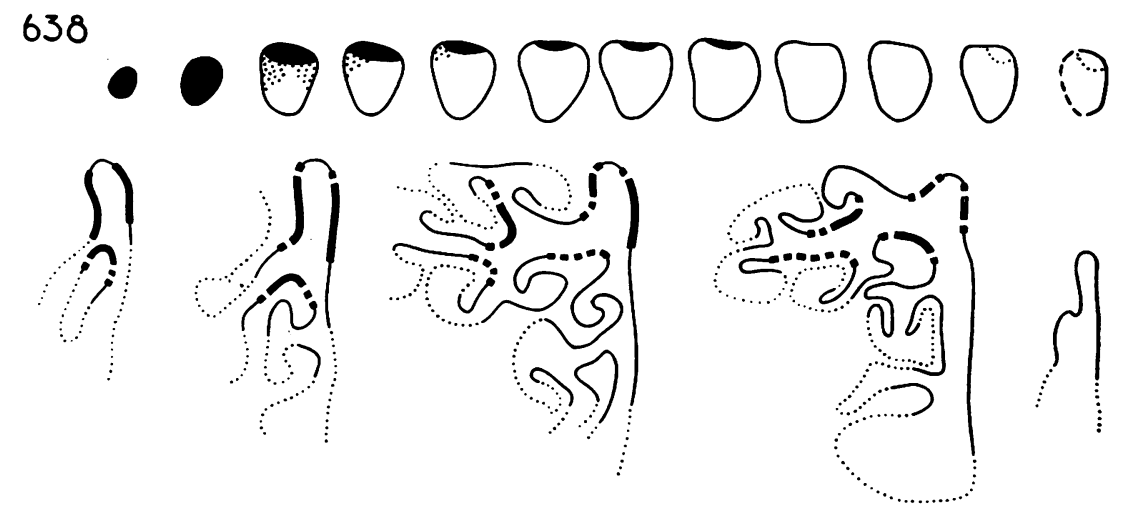

FIG. 6.-Diagram illustrating experiment R.638. 


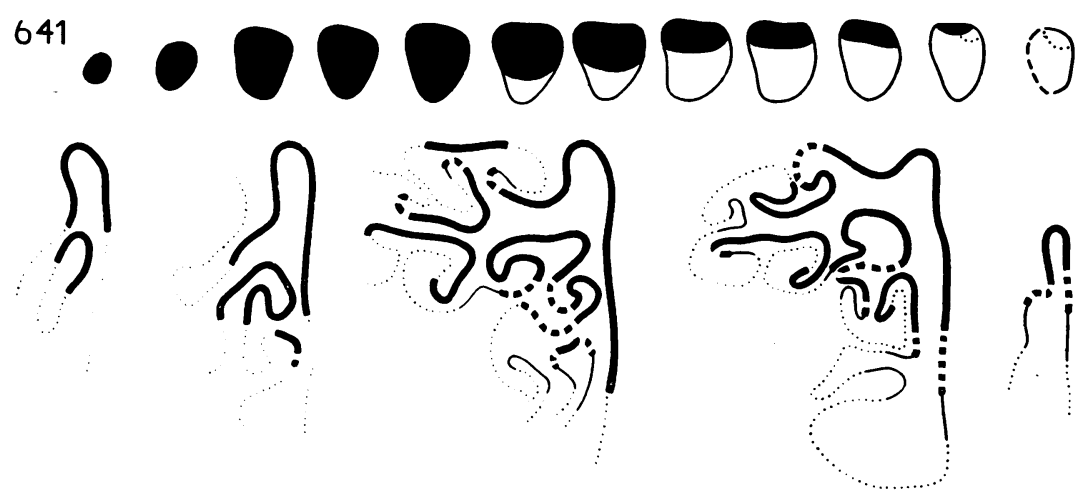

FIG. 7.-Diagram illustrating experiment R.641.

In Figs. 3-8 areas of severe degeneration of the olfactory epithelium are indicated by a continuous thick line, and of moderate degeneration by an interrupted thick line. Areas of apparently normal (or nearly normal) epithelium are indicated by a continuous thin line. Dotted lines represent the non-olfactory part of the nasal mucous membrane.

Twelve transverse sections of the olfactory bulb, taken at regular intervals, are represented above. The extent of total destruction is indicated in black and areas of partial involvement in stipple. In all cases the lateral aspect of the bulb is shown to the left. Below are shown, from left to right, outlines of transverse sections taken at regular antero-posterior intervals through the nasal cavity of the affected side, the septa epithelium in each case being shown to the right.

on the first endoturbinal, presumably in relation to the fact that the anterior extremity of the olfactory bulb had been completely destroyed.

Experiment R.641 (Fig. 7).-At the operation a lesion was made involving the anterior two-thirds of one olfactory bulb, and the animal was killed six weeks later. Serial sections show that approximately the anterior half of the bulb had been completely removed, while in the posterior half a corsiderable proportion of the dorsal glomeruli had been destroyed.

The olfactory epithelium shows an extensive distribution of severe degeneration. This involves the whole of the septal epithelium (with the exception of the most ventral portion posteriorly), practically the whole length of the first and second endoturbinals (except along their attached margins where the degeneration is moderate), the roof of the lateral nasal recess, the upper and lower ectoturbinals, and almost the entire surface of the nasoturbinal. The epithelium of the third endoturbinal shows degeneration over its upper surface. That covering the fourth endoturbinal and the small middle ectoturbinal appears to be intact.

A careful study of the olfactory epithelium where it appears unaffected, for example, at the lower margin of the posterior end of the septal area, and over the fourth endoturbinal, with special reference to its relative depth, its cell density, and histological traces of cytolysis, fails to show evidence of even a partial degeneration. It seems probable, therefore, that these areas project entirely, or almost entirely, on to the ventral part of the posterior half of the bulb. This evidence for a form of localized projection, based on the connexions of a relatively small region of the bulb left intact after destruction of the greater part, is perhaps more convincing than in those experiments in which the bulbar lesion has been of very limited extent. For in the latter experiments it is not easy to assess the amount of indirect involvement of glomerular connexions beyond the immediate boundary of the primary lesion.

Experiment R.642 (Fig. 8).--In this experiment one bulb was almost completely destroyed except for the medio-ventral part of its posterior third. The animal was killed six weeks after the operation. Serial sections show that even those glomeruli spared by the main lesion are not all intact, for in the neighbourhood of the lesion many are grossly shrunken, presumably as the result of a partial or complete interruption of their olfactory fibre connexions.

Almost the whole extent of the olfactory epithelium shows severe degeneration. The only region which shows no evidence of degenerative changes is the lower margin of the septal area and the lateral wall of the nasal cavity at the extreme posterior end of the latter, and a small strip on the under surface of the third endoturbinal. A moderate degree of degeneration affects the lower margin of the septal area in the posterior

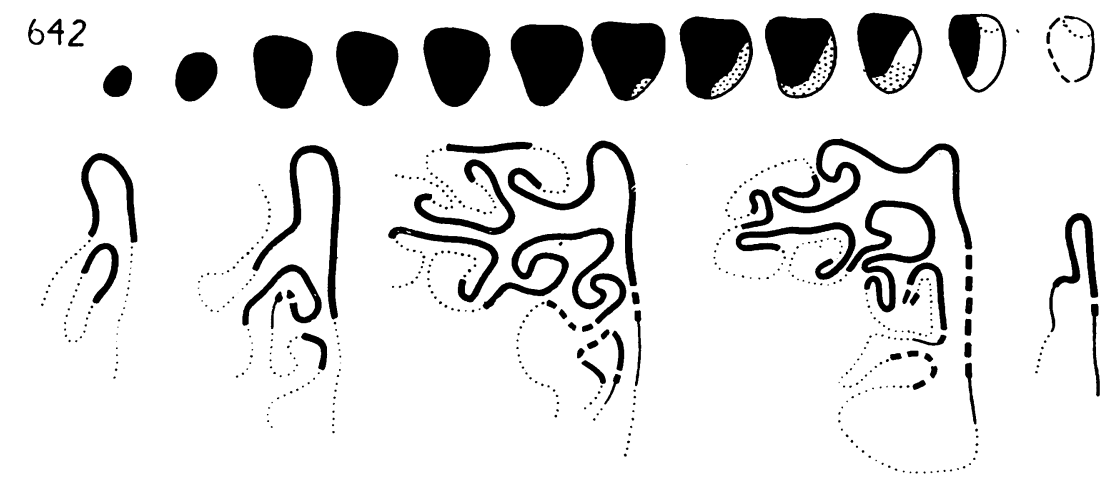

FIG. 8.-Diagram illustrating experiment R.642. 
half of its extent, the lower surface of the second endoturbinal, the upper lip of the third endoturbinal, and the epithelium covering the fourth endoturbinal. The distribution of the epithelial degeneration is thus similar to that of the previous experiment (R.641) but rather more extensive.

Experiment R.644. - The lesion in this case was similar to that of the previous experiment (R.642). The animal was killed six weeks after the operation, and serial sections of the bulb show complete destruction except for the medially situated glomeruli in the posterior third of the bulb.

The serial sections through the nasal cavity are not entirely satisfactory, for the mucous membrane is affected by scattered patches of atrophic rhinitis. However, where the epithelium appears normal in structure it provides some reliable information regarding the distribution of secondary degeneration following the bulbar lesion. The extent of this degeneration corresponds closely with that in experiment R.642. It involves the whole of the septal epithelium, except the ventral part of its most posterior portion. The epithelium covering the medial aspect of the third and fourth endoturbinals also appears to be intact. On the other hand, the epithelium of the lateral wall of the nasal cavity has undergone severe degeneration throughout its whole antero-posterior extent.

\section{Discussion}

It is quite evident, from the results of the experiments recorded above, that there is some degree of spatial localization in the olfactory bulb in the sense that the upper areas of the olfactory epithelium project mainly on to the upper part of the bulb, and vice versa. The experiments also provide some evidence that the more anterior and posterior areas tend to project on to the more anterior and posterior regions of the bulb respectively, but here the relationship appears to be more diffuse. In experiment L.1, for example, where the posterior part of the bulb had been left intact, severe degeneration of the epithelium was found to extend back to the posterior extremity of the dorsal recess, while in experiments R.146 and R.637, where the anterior part of the bulb had escaped the lesion, severe degeneration extended to the anterior extremity of the dorsal recess and the nasoturbinal. In a mediolateral direction also, the topographical projection of the olfactory epithelium on to the bulb seems to be not very sharply defined, though the experiments again provide evidence for a regional projection of some degree in this direction.

If the projection of different areas of the olfactory epithelium is considered separately, the following general statements appear to be justified by the experiments. The dorsal part of the septal epithelium projects mainly on to the dorsally situated glomeruli, and the more ventral part to those situated medially and ventrally. The epithelium covering the nasoturbinal likewise projects largely on to the dorsal glomeruli, though there is some evidence that the more lateral region of this epithelium is predominantly related to the glomeruli on the lateral surface of the bulb near its upper margin. The epithelium covering the dorsal surfaces of the first and second endoturbinals (particularly the former) appears to be most directly related to the front and upper parts of the bulb, while that covering the less exposed areas of these processes only undergoes degeneration

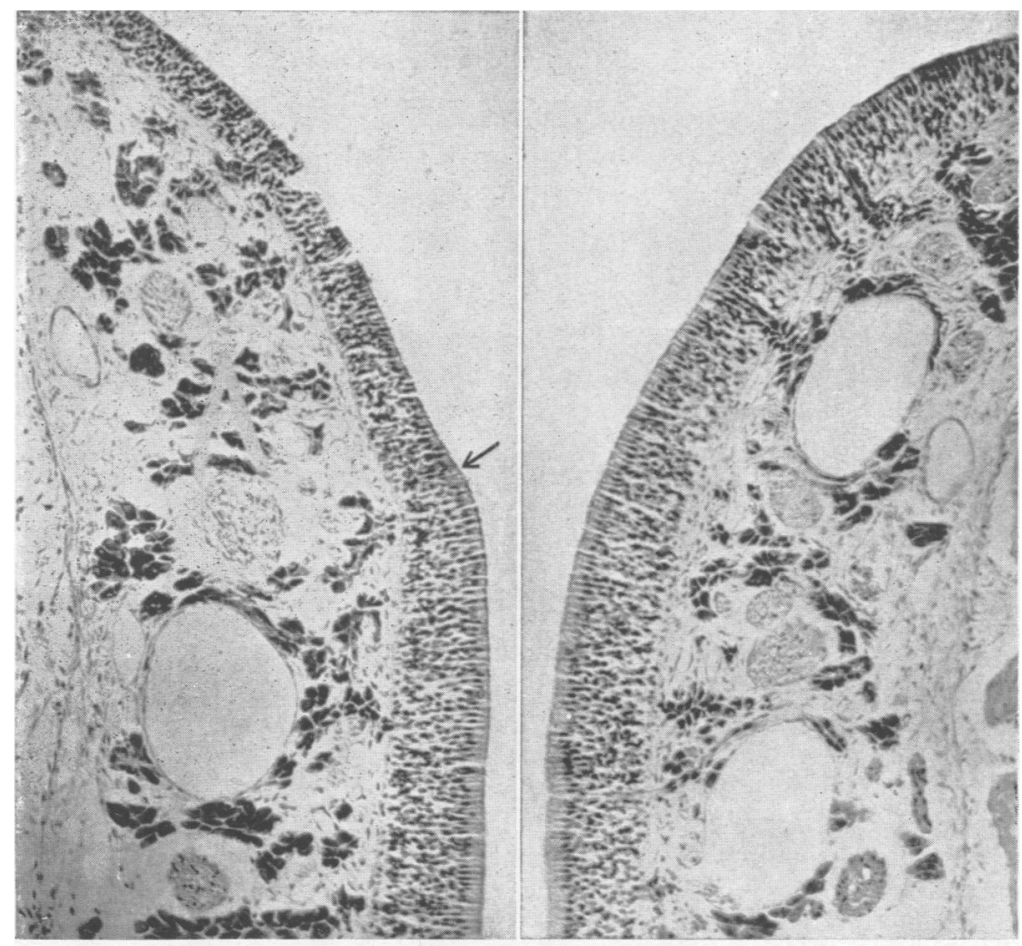

FIG. 9.-Sections of the olfactory epithelium covering the first endoturbinal in experiment R.146 on the operated side $(a)$ and the normal side $\left(b^{\prime}\right.$. $(\times 120$.) On the operated side the arrow marks the abrupt transition between apparently normal epithelium and severely degenerated epithelium. 
as the result of extensive lesions involving the more ventral and posterior regions of the bulb. The epithelium of the third and fourth endoturbinals is evidently related predominantly to the glomeruli in the most postero-ventral part of the bulb. As regards the epithelium covering the ectoturbinals in the lateral recess of the nasal cavity, the experiments suggest that the more exposed parts tend to project on to the more dorsal glomeruli, and the less exposed parts on to ventral and lateral glomeruli.

It is not possible to draw more precise conclusions on the topographical localization in the olfactory bulb from these experiments, because the material only permits an approximate delineation of the extent of the lesion, complete or partial, affecting different glomeruli; nor does it afford a very exact quantitative evaluation of the degree of secondary degeneration affecting the receptors in different parts of the olfactory epithelium. In general, however, the type of localization which has been demonstrated accords reasonably well with that which has been reported by Adrian on the basis of electro-physiological studies. That the projection, in relation to at least some areas of epithelium, may perhaps be more precise than the present series of experiments indicates, is suggested by the observation that in certain cases of partial lesions of the bulb the transition between apparently normal and severely degenerated areas of epithelium is rather abrupt. This appearance is shown in Fig. 9, in which the olfactory epithelium covering the first endoturbinal in experiment R.146 is seen on the operated and normal sides. In this particular case it is difficult to explain the abruptness of the transition between the two epithelial areas except on the assumption that the junctional margin of the corresponding projection areas in the olfactory bulb is equally well defined.

That there is no regular point-to-point projection of the olfactory epithelium on to the bulb, in the sense that, as with the projection of the retina on to the lateral geniculate nucleus, each local area of the sensory epithelium is represented centrally in an orderly sequence of equivalent areas having the same spatial relationship, seems assured by a histological study of normal material. It is now established that the olfactory fibres derived from the receptors in the olfactory epithelium pass directly up to the cribriform plate in parallel fasciculi, and without any significant rearrangement while they are in the nasal cavity. But as soon as they penetrate the cribriform plate to enter the cranial cavity, the individual fasciculi split up into smaller components and the latter become involved in a most complicated plexus of interweaving fibres on the surface of the olfactory bulb before they enter the latter. It appears certain, therefore, that the spatial relationship of the nerve fibres within the nasal cavity is by no means preserved up to the actual site of their entrance into the bulb. For example, in protargol-treated sections, some fibres approaching the anterior tip of the bulb from a more ventral direction in the nasal cavity may be seen crossing those coming from a more dorsal direction so as to reach the dorsal aspect of the bulb, and vice versa. An examination of individual glomeruli, also, shows olfactory fibres approaching and entering them

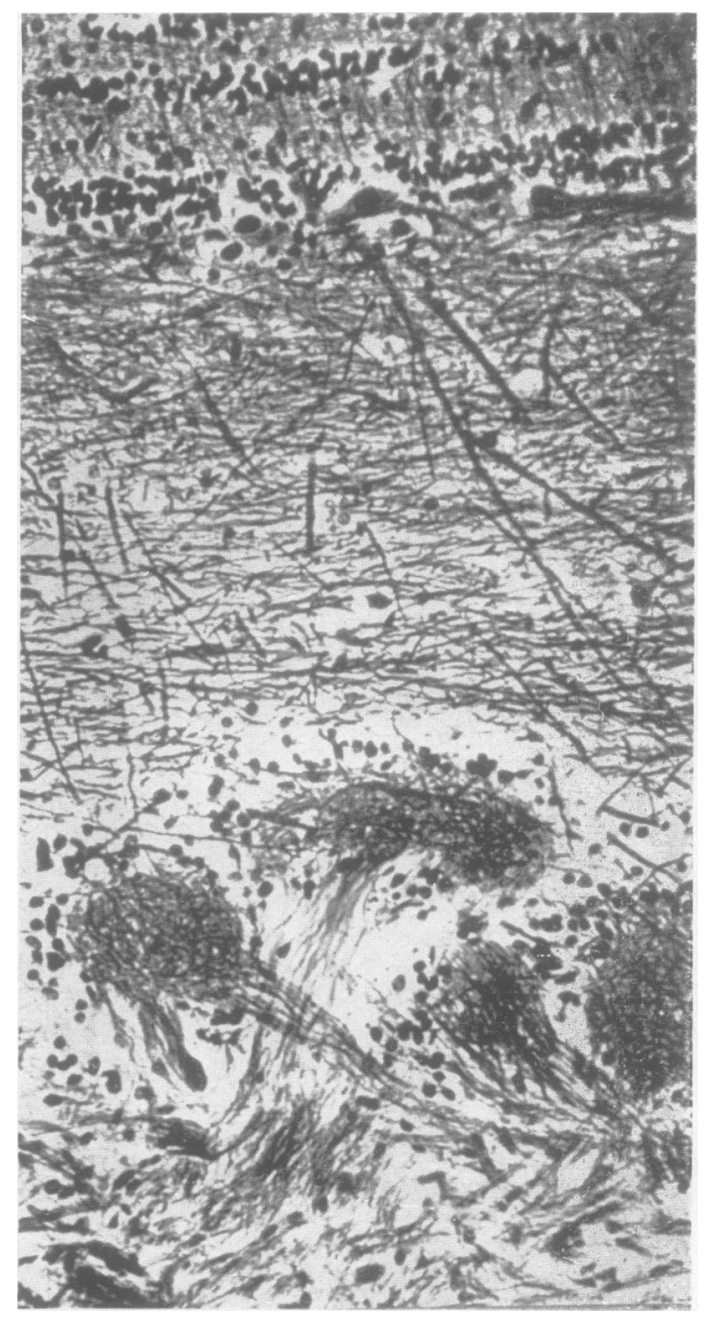

FIG. 10.-Section of a normal olfactory bulb of a rabbit stained with protargol, showing adjacent glomeruli receiving bundles of olfactory fibres entering from different directions. Above the glomeruli is seen the outer molecular layer of the bulb, traversed by the dendrites of mitral cells. ( $\times 210$.) 
from many different directions. Two such examples are shown in Figs. 10 and 11, and in the latter the intricacy of the plexus of olfactory fibres on the surface of the bulb is made very apparent. Clearly, this plexiform arrangement is the occasion for a resorting of olfactory fibres which makes it possible for some or all the glomeruli to receive impulses from different areas of the olfactory epithelium not necessarily in direct topographical relationship to each other, and it may be surmised that this is in some Wway associated with functions of olfactory discrimination. One possibility is that there are different types of olfactory receptor which are differentially susceptible to various forms of olfactory stimulus, and that impulses from the several categories of receptor become segregated and conveyed to different glomeruli of corresponding categories. On the other hand, as Adrian has

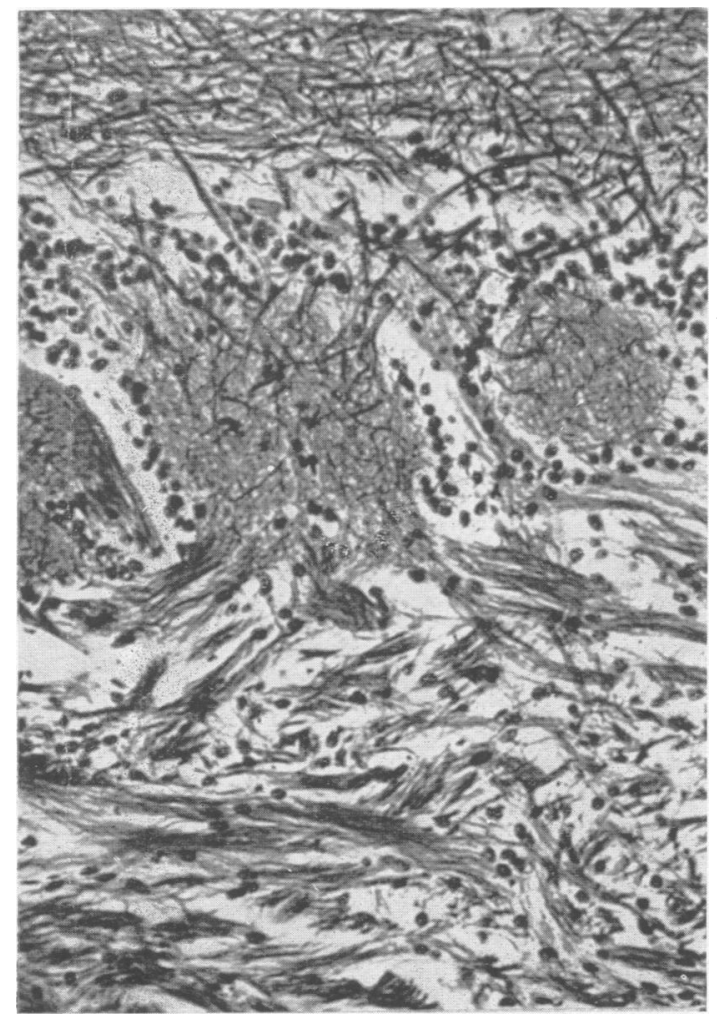

FIG. 11.-Section of a normal olfactory bulb of a rabbit stained with protargol showing a glomerulus receiving olfactory fibres which enter it from opposite directions. Below the glomerular layer is seen part of the complicated plexus of olfactory fibres on the surface of the bulb. Entering the large glomerulus from above are seen numerous dendrites of mitral cells. $(\times 210$. pointed out, there is no evidence as yet that the olfactory epithelium is composed of anything like a uniform mosaic of different kinds of receptor each with specific affinities for certain kinds of odoriferous substances, though the possibility of some such organization is not necessarily precluded by the methods at present available for the analysis of olfactory impulses reaching the bulb. Another possible interpretation of the resorting of olfactory nerve fibres is that those derived from equivalent regions of the complicated turbinal system (for example, from the more exposed surfaces or from the deeper and less immediately accessible recesses) are regrouped so as to be led to different sets of glomeruli. Such an arrangement would be consistent with Adrian's suggestion that olfactory discrimination may depend, at least in part, on a regional excitation of the olfactory epithelium by the differential diffusion of odoriferous substances of varied molecular size. This suggestion involves the consideration that the complicated system of turbinate processes in animals such as the rabbit is not simply a device for increasing the surface extent of the olfactory epithelium, but may be an important factor in determining the direction followed by odoriferous particles having differential rates of diffusion, so as to lead to specific spatial patterns of stimulation of the epithelium. This hypothesis is particularly attractive since it eliminates the need for postulating the existence of olfactory receptors of many different types. However, one theoretical difficulty which presents itself is that an intricate folding of the olfactory epithelium itself is evidently not an essential prerequisite for olfactory discrimination. In man, for example, the olfactory epithelium is not involved in the labyrinthine convolutions found in most lower mammals, for it is here represented by two approximately flat sheets covering the upper third of the septum and the medial surface of the superior concha. Yet man is capable of an astonishingly high degree of olfactory discrimination, even though his olfactory sensitivity may be considerably less than that of lower mammals. It is possible that olfactory discrimination depends on several different factors, of which a spatial pattern of stimulation of the olfactory epithelium is but one. In any case, whatever the peripheral analytical mechanism for olfactory discrimination may be, on anatomical grounds the glomeruli of the olfactory bulb appear to provide a suitable basis as a series of central analysers, for, apart from the fact that some of the small periglomerular cells may permit a certain degree of functional interaction between immediately adjacent glomeruli, each glomerulus with its connecting mitral cells forms virtually a closed system. 
Thus, through the individual glomeruli separate groups of impulses from the olfactory epithelium may be segregated as far as their termination in the prepiriform cortex and adjacent regions of the cerebral hemisphere.

It would clearly be desirable, in connexion with the problem of olfactory discrimination, to obtain much more precise information regarding spatial localization in the olfactory bulb than has been possible with the methods used in the experiments recorded here. This could no doubt be done by effecting more sharply circumscribed lesions limited to localized groups of glomeruli (using such methods as thermocoagulation), and defining more precisely the degree and extent of the ensuing degeneration in the olfactory epithelium by making accurate counts of individual receptors in silverimpregnated preparations. Technically this presents a somewhat formidable task, but it should make it possible to determine just how far the localized projection of different areas of the olfactory epithelium on to the olfactory bulb is theoretically capable of providing a basis for olfactory discrimination.

\section{Summary}

The distribution of secondary degeneration in the olfactory epithelium following lesions of the olfactory bulb has been studied in a number of rabbits.

The experiments show that there is some degree of spatial localization in the projection of different areas of the olfactory epithelium on to different regions of the bulb.

The evidence suggests that, although the projection as a whole is of a somewhat diffuse type, in regard to some areas of the olfactory epithelium it may be rather precise.

\section{REFERENCES}

Adrian, E. D.(1950). Brit. med. Bull., 6, 330. (No. 1534).

Le Gros Clark, W. E., and Warwick, R. T. T. (1946). Journal of Neurology, Neurosurgery and Psychiatry, 9,101 . 